\title{
EXPERIMENTAL AND NUMERICAL ANALYSIS OF CAVITATING FLOW AROUND A HYDROFOIL
}

\begin{abstract}
Milan SEDLÁR ${ }^{(1)}$, Martin KOMÁREK ${ }^{(1)}$, Michal VYROUBAL $^{(1)}$, Miloš MÜLLER $^{(2)}$ •
Abstract: The paper describes experiments carried out in the cavitation tunnel with the rectangular test section of $150 \times 150 \times 500 \mathrm{~mm}$ and the maximum test section inlet velocity of $25 \mathrm{~m} / \mathrm{s}$. These experiments have been aimed to visualize the cavitation phenomena as well as to quantify the erosion potential using pitting tests evaluated during the incubation period for the cast-iron prismatic hydrofoil with the modified NACA profile. A bypass section installed in the tunnel has allowed to measure the nuclei content in the inlet flow to the test section using the acoustic spectrometer. The measured data have been compared with the CFD analysis of the cavitation phenomena on the hydrofoil as well as the numerically determined location and magnitude of the first calculated collapses of the cavitating bubbles with a good agreement.
\end{abstract}

\section{INTRODUCTION}

Cavitation is a phenomenon of many faces. As a tool it is used in many industrial, biochemical or medical processes and applications. Unfortunately, in hydromachinery cavitation usually causes negative effects, such as the degradation of the machine performance, the cavitation surge which can generate unwanted noise and vibrations or the material erosion. The cavitation analysis became a part of widespread CFD packages and it is often successfully used to model the adverse effect of cavitation in hydraulic machinery. According to our long term experience with the ANSYS CFX software, very good results can be obtained when modelling cavitation in the blade passages, wearing rings or in the tip clearance gap and tip vortex, as well as the degradation of the machine performance (e.g. [1 - 2]). We also tried to model some cavitation instabilities using this commercial code with fairly encouraging results [3 - 4]. We see that the problems in this area are more related to the modelling of turbulence than to the cavitation models.

A bit different situation is in the case of modelling the cavitation erosion. For example, the Rayleigh-Plesset model built in the ANSYS CFX and Fluent packages describes the time derivative of the radius of the spherical cavitation bubble with a simplified RayleighPlesset equation, in which the higher order terms are neglected. This simplification results in the impossibility to predict the rebounds of the cavitation bubbles as well as the position and energy of the bubble collapses. The cavitating flow behaviour is also sensitive to the physical properties of the liquid, especially to the nuclei content.

\footnotetext{
- (1) SIGMA Research and Development Institute, Jana Sigmunda 79, 78350 Lutín, milan.sedlar@sigma-vvu.cz, martin.komarek@sigma-vvu.cz, michal.vyroubal@sigma-vvu.cz

(2) Faculty of Mechanical Engineering, Technical University of Liberec, Hálkova 6, 46117 Liberec, milos.muller@tul.cz
} 
Nevertheless in the CFD applications the nuclei size distribution is typically neglected or highly simplified (e.g. the previously mentioned Rayleigh-Plesset model considers only one size of the initial nuclei radius).

The SIGMA Research and Development Institute has been interested in the development of a suitable cavitation erosion model for many years, cooperating in this field with the Institute of Thermomechanics of the Academy of Sciences of the Czech Republic as well as the Technical University of Liberec. This cavitation erosion model is based on the twoway coupling of the CFD analysis of 3D turbulent flow with the modelling of the cavitation bubble dynamics along selected streamlines. The CFD part uses the in-house software developed in the SIGMA Research and Development Institute. The bubble dynamics is based on the solution of full Rayleigh-Plesset equation and considers that the water running through the hydraulic machinery parts contains a known number of air-filled or vapour-filled microbubbles of the known size distribution. The number and size of the initial cavitation nuclei are predicted empirically or experimentally. To be able to validate the cavitation erosion model, the SIGMA Research and Development Institute built a water tunnel, which has been used for several projects concerning the visualization of the cavitation phenomena as well as the cavitation erosion tests.

\section{Cavitation tunnel}

The cavitation tunnel was built and put into operation in 2007 and represents a closed, horizontal plane water tunnel for isolated hydrofoils (Fig. $1-2$ ). The capacity of the main tank is $35 \mathrm{~m}^{3}$. The closed loop is equipped with both the compressor and the vacuum pump capable of creating different pressure levels while maintaining constant volume flow rate. To maintain the quality of water and to remove the solid particles which could affect the heterogenous nucleation in the water during the tests, the filtration unit was added in the bypass between the end of the discharge piping and the main tank. The filter is able to treat the particles with the minimum size of $10 \mu \mathrm{m}$. The flow rate through the filtration unit is $14 \mathrm{~m}^{3} / \mathrm{h}$ (3.9 LPS).

The rectangular test section (Fig. 3) has the inner dimensions $150 \times 150 \times 500 \mathrm{~mm}$, which corresponds to the test section inlet velocity of $25 \mathrm{~m} / \mathrm{s}$ for the maximum flow rate of the variable-speed driven axial-flow pump. The transit time of one fluid particle resulting simply from a complete tunnel loop capacity is $72 \mathrm{~s}$ for the maximum flow rate.
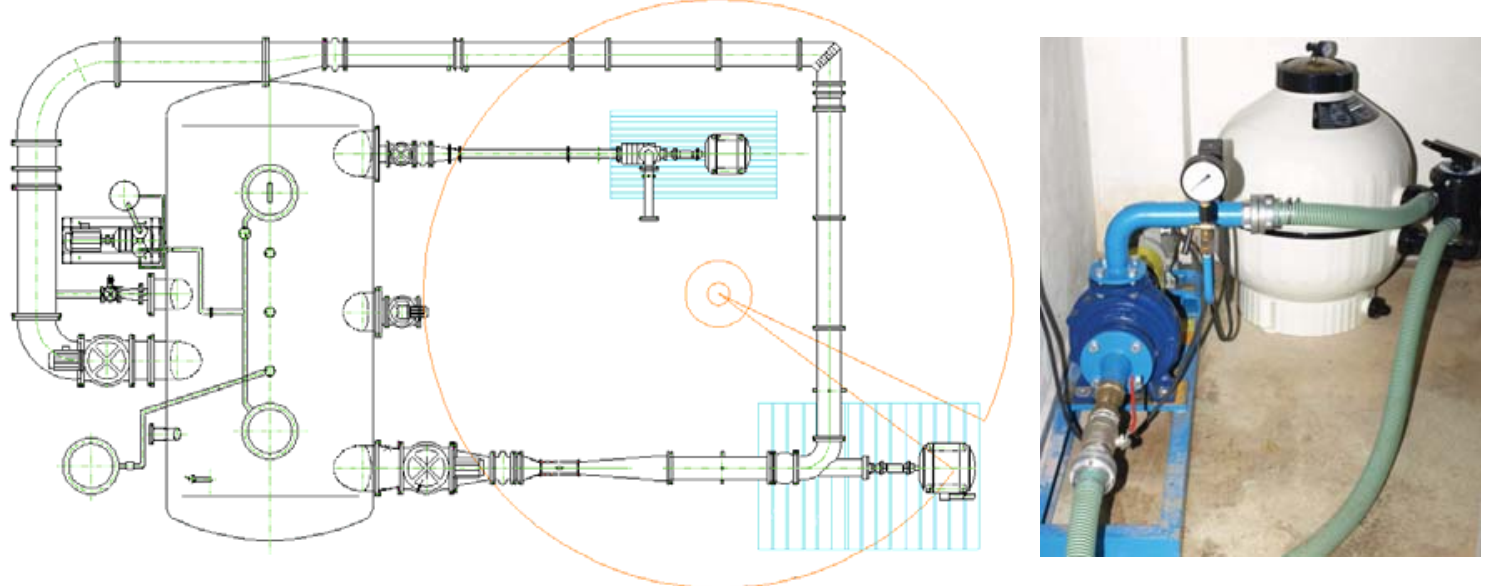

Figure 1: Scheme of whole horizontal loop and detail of filtration unit 


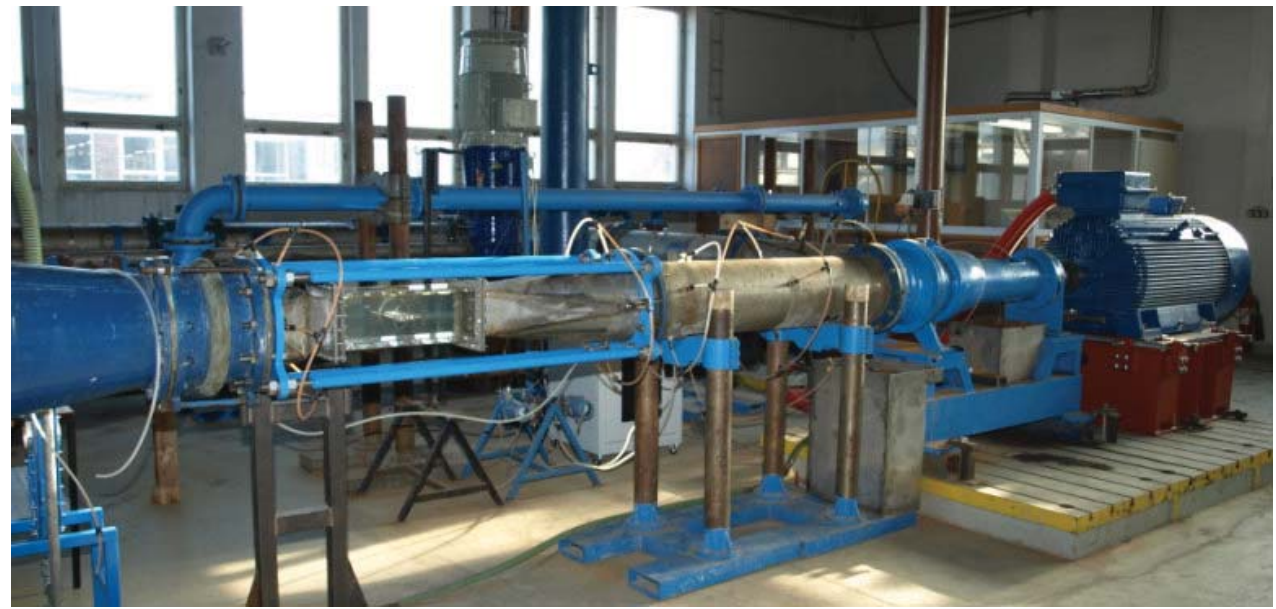

Figure 2: Cavitation tunnel

At the test section inlet, different types of honeycombs can be added. As the crosssection of the tunnel changes from the circular one (piping DN300) to the square one at the test section and back, the contraction chamber and the diffuser were designed and optimised using the CFD tools. The hydraulic surfaces of these parts were carefully machined and polished. As there is a cone reduction of the piping from the diameter of $500 \mathrm{~mm}$ to the diameter of $300 \mathrm{~mm}$ in front of the contraction chamber, the total contraction ratio of 8.7 is achieved. The velocity uniformity at the front part of the test section is (based on the CFD analysis) within $\pm 1 \%$, excluding the boundary layer.

The currently tested hydrofoils are prismatic with a typical chord length of $100 \mathrm{~mm}$ and a maximum span of $150 \mathrm{~mm}$ (corresponding to the width of the test section). The profile incidence angle $\alpha$ can vary between $0^{\circ}$ and $\pm 180^{\circ}$. The test section is made of the organic glass to facilitate visualizations. The experimental 2D hydrofoil, shown in Fig. 3, has a maximum thickness of $23.5 \mathrm{~mm}$. Its cross-section consists of the $3: 2$ elliptical nose and the NACA 0020 tail joined at the maximum thickness.
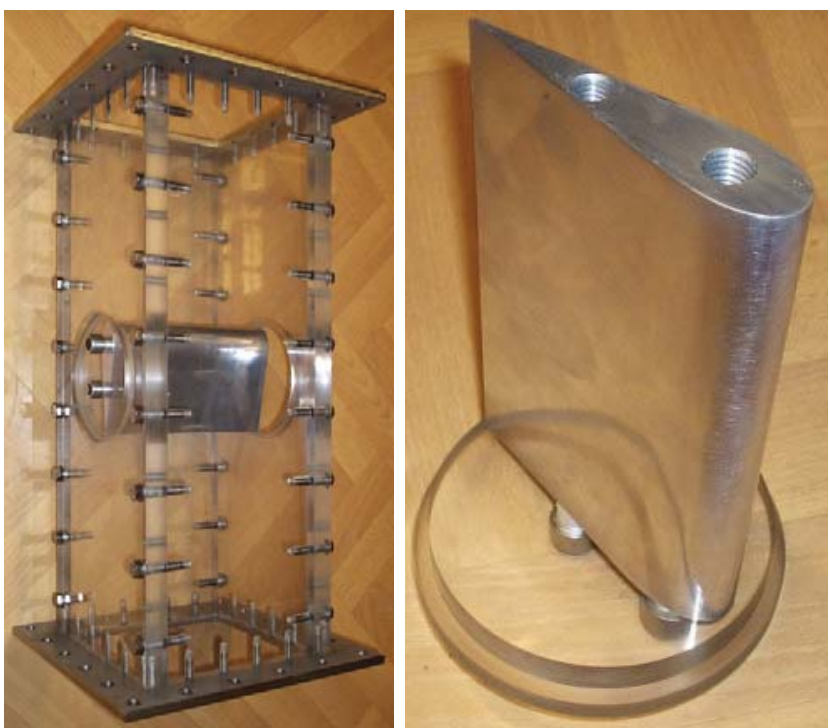

Figure 3: Test section and 2D hydrofoil

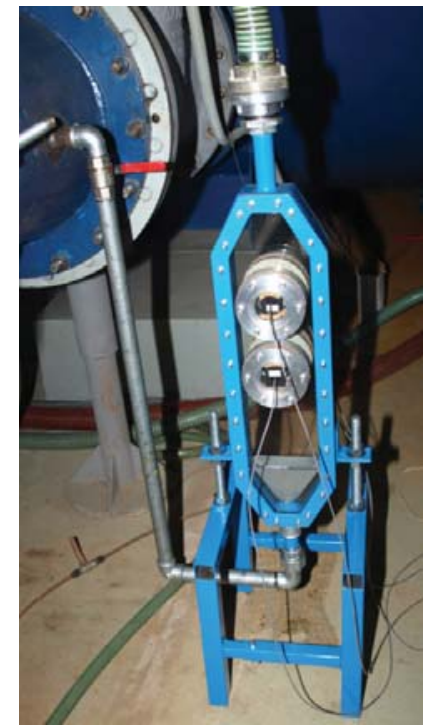

Figure 4: Acoustic spectrometer 
To measure the volume flow rate, the magnetic-inductive flowmeter is applied. It works in the accuracy class of $0.3 \%$. For all measurements of the pressure differences, the transducers of the accuracy class $0.1 \%$ are used. The temperature of the water is measured with the platinum thermometer in the class of accuracy $0.5 \%$. To enable monitoring of the cavitation nuclei in the inlet flow, a bypass section is installed allowing to measure the nuclei content using the ABS acoustic bubble spectrometer (Fig. 4). In cooperation with the Technical University of Liberec we can use two sets of hydrophones - $50 \mathrm{kHz}$ and $150 \mathrm{kHz}$ ones - which enable us to measure the bubble radii between 25 and 105 micrometers.

\section{Cavitation EROSION MOdel AND CFD tOOLS}

As mentioned in the introduction the cavitation erosion model is based on the two-way coupling of the CFD analysis of 3D turbulent flow with the modelling of the cavitation bubble dynamics along selected streamlines (Euler - Lagrange approach). The CFD part uses the in-house software developed in the SIGMA Research and Development Institute. This is a FEM-based solver of the 3D RANS equations, which applies the generalised form of the penalty formulation to take into account the density changes in the cavitating zones [5]. In the bubble dynamics model we apply full Rayleigh-Plesset equation. Because only the bubbles with a gas content can reach more collapses, it is assumed that the bubble interior is formed by a mixture of the water vapour and the non-condensable gases [ $6-8]$. In the presented calculations the isothermal behaviour of the gas mixture is supposed. The Rayleigh-Plesset equation is solved by the Runge-Kutta fourth-order scheme with the adaptive step-size algorithm.

The modelling of the erosion effects starts from the assumption that the main contribution to the erosion of solid materials arises from the mechanical effects of the collapsing bubble near the solid surface. The erosion potential of the collapse is estimated from the energy dissipated during this collapse [9 - 10]. The effect of the liquid viscosity, compressibility and surface tension is neglected in the present model. The dissipated energy is evaluated for each bubble in the ensemble of bubbles of different initial sizes and for its each individual collapse along the streamlines nearest to the solid surface.
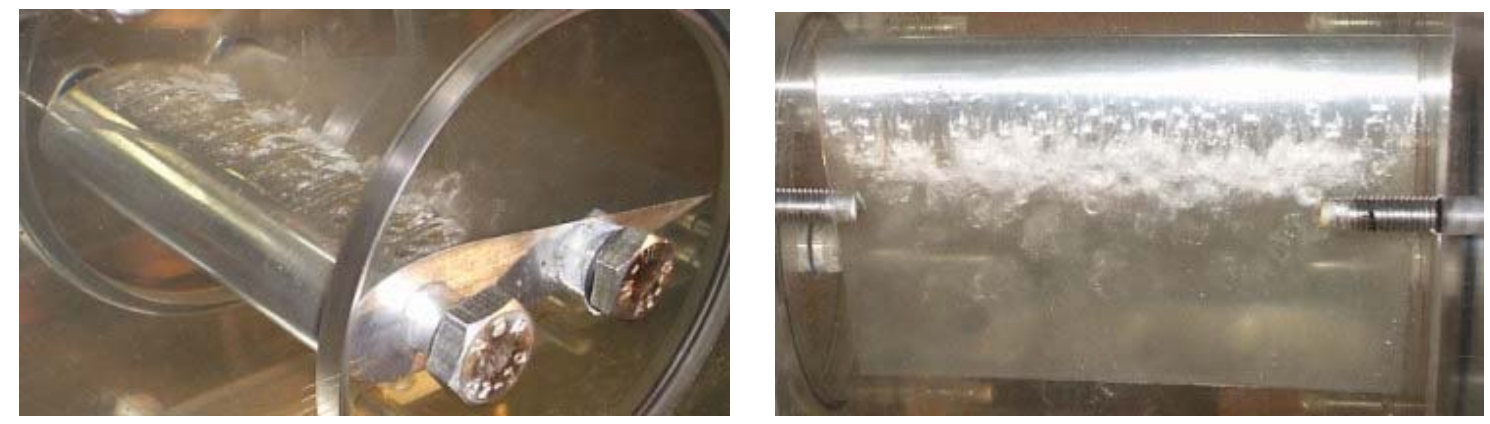

Figure 5: Uniform shape of cavitation region during steady attached cavitating flow. Experiment, $\alpha=0^{\circ}, \sigma=1.9$

To model the cavitation inside the hydraulic machine resulting in the degradation of the machine performance or to model the cavitation surge we use the ANSYS CFX commercial CFD package. It applies the Rayleigh-Plesset model, which is included in 
the ANSYS CFD software to describe the interphase mass transfer in the framework of the homogenous multiphase model. The two-phase system is solved for the vapour and the liquid with a non-condensable gas. Though this cavitation model is based on a highly simplified Rayleigh-Plesset equation for the radius of a spherical cavitation bubble, it is very robust and effective for the analysis of the problems, where the void fraction is highly important in forming flow structures but where the details of the bubble dynamics (especially the collapses and rebounds) are not of primary interest [2 - 4]. To capture the highly unsteady phenomena we usually use the SST-SAS turbulence model. It represents a good compromise between the widely used SST model and the LES or DES models, which are too costly for the comprehensive turbomachinery applications [11].
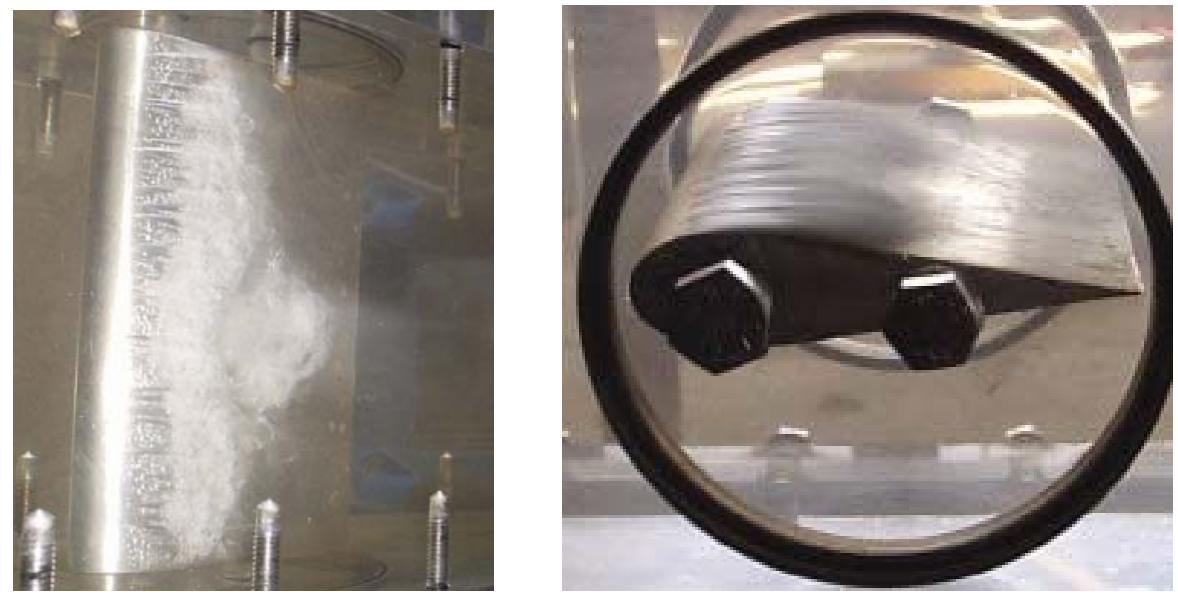

Figure 6: Partial cavity oscillation. Experiment, $\alpha=0^{\circ}, \sigma=1.8$

\section{VISUALIZATION OF CAVITATION PHENOMENA ON 2D BLADE}

An extensive tunnel testing has been performed to verify the tunnel functionality for a wide range of flow regimes and to compare the observations with the preliminary numerical analysis. First, the regimes with the attached partial cavitation were tested with the incidence angle $\alpha=0^{\circ}$. It was found that for relatively high cavitation numbers $(\sigma \geq 1.85)$, the attached partial cavity is relatively steady and has a shape of the stripe, which is equally wide in the spanwise direction (Fig. 5). With decreasing cavitation number $\sigma$, the cavitation region becomes unsteady (so called partial cavity oscillation) and changes its length and shape (from the U shape to the W shape - Fig. 6). When the cavitation number drops below the value of 1.7 , the supercavitation regime starts, with the cavitation bubbles collapsing behind the trailing edge (Fig. 7). This regime is unsteady up to the cavitation number about 1.5 , when the steady stable supercavitation region forms with a sharp interface between the water and a wake filled with the cavitation bubbles (Fig. 8). Because our main interest was to find the steady regime for the pitting tests, the frequency analysis of the unsteady cavitation phenomena was not done due to lack of suitable high-speed camera. Nevertheless the partial cavity oscillation was simulated with the ANSYS CFX tool for the cavitation number of 1.8 (Fig. 9) with the obtained Strouhal number approximately St $=0.2$, which is somewhere between the values obtained by Watanabe et al. ([12]) for the partial and transitional cavity oscillations. As far as the supercavitation regime is concerned, only a steady regime with sufficiently low cavitation number was modelled successfully with the CFD tools (Fig. 10). 

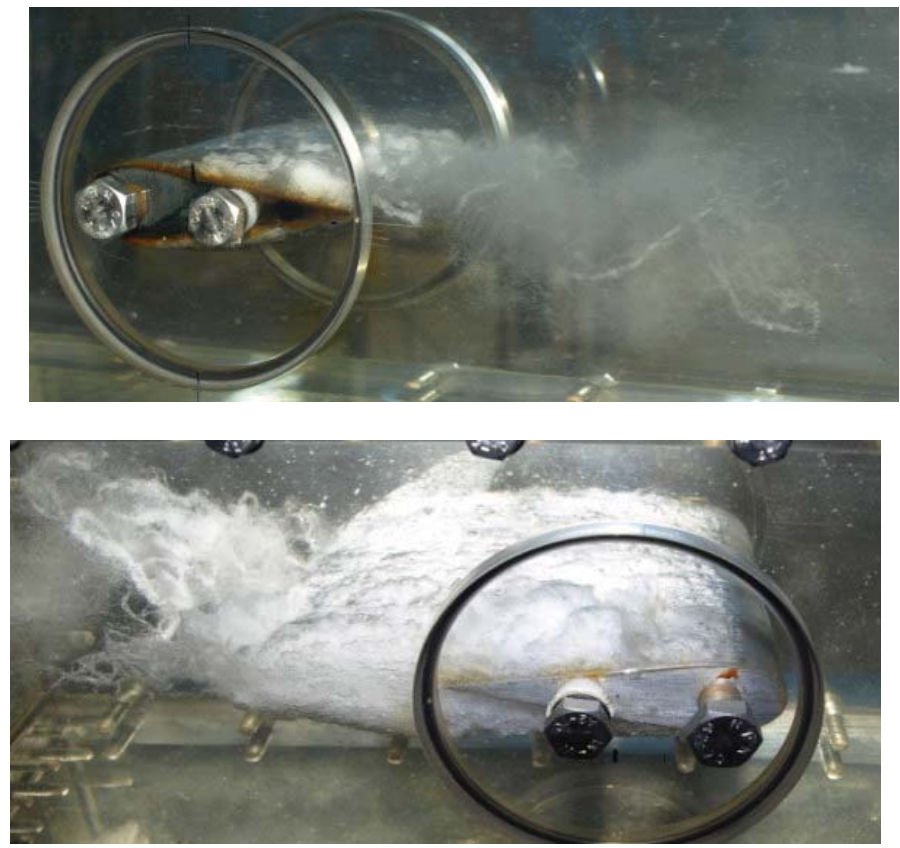

Figure 7: Unsteady supercavitation regimes. Experiment, $\alpha=0^{\circ}, \sigma=1.7-1.6$

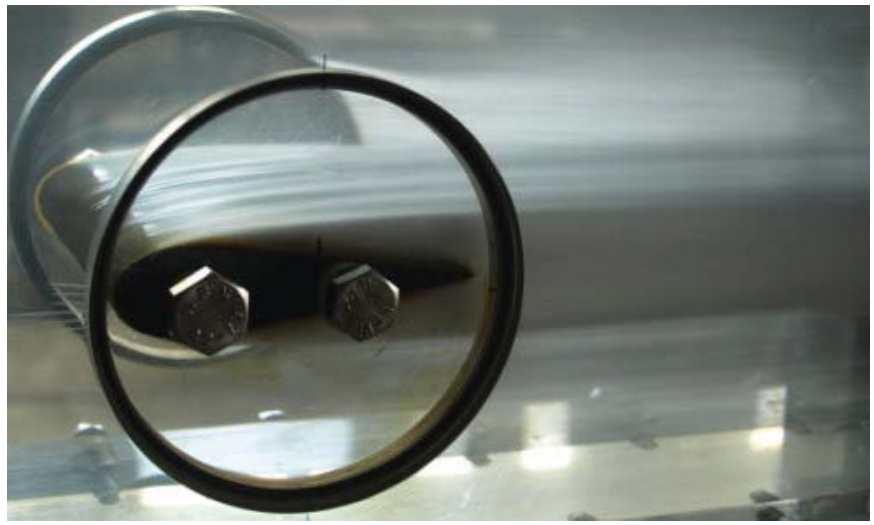

Figure 8: Steady supercavitation regime. Experiment, long time exposure, $\alpha=0^{\circ}, \sigma=1.5$

\section{EXPERIMENTAL AND NUMERICAL ANALYSIS OF CAVITATION EROSION ON 2D BLADE}

As mentioned in the introduction the main objective of the experimental research in the cavitation tunnel was to validate the cavitation erosion model developed in cooperation of the SIGMA Research and Development Institute with the Institute of Thermomechanics of the Academy of Sciences of the Czech Republic and the Technical University of Liberec. That is why the pitting tests were carried out for a sufficiently long time interval to describe the incubation period of the cavitation erosion. The incidence angle of $-1^{\circ}$ was chosen to get two partial cavities with different length on the suction and pressure sides of the hydrofoil. To avoid the unsteady cavitation regime, the cavitation number $\sigma=1.93$ was set. Figure 11 shows individual cavitation bubbles on the upper (pressure) side of the hydrofoil just before their collapse, and a very narrow stripe of pits on the same hydrofoil side. In Fig. 12 the detail of pits of different sizes on the pressure side can be seen after 63 and 120 hours of the pitting tests. Fig.13 shows the size distribution of pits 
in the cast iron hydrofoil after 63 hours of the pitting tests and the repeatability of the measurements. In cooperation with the Technical University of Liberec we used two sets of hydrophones $-50 \mathrm{kHz}$ and $150 \mathrm{kHz}$ ones - which enabled us to measure the bubble radii between 25 and 105 micrometers. Two tests are presented in Fig.14 (denoted as Sigma1 and Sigma2), each with 300 sample measurements. For reference, the measured bubble spectra are compared with the spectrum obtained by Waniewski et al. [13]. We used measured initial bubble size distribution with some correction. As the most important nuclei - from the point of cavitation erosion - can be found usually in the range from 10 to 100 micrometers, we extrapolated the distribution curve to the left. In the area with a drop of the bubble numbers (55 - 75 micrometers) we interpolated the curve as we suppose that the drop is caused by the fact that the effective ranges of both hydrophones do not overlap and there is a low level signal.
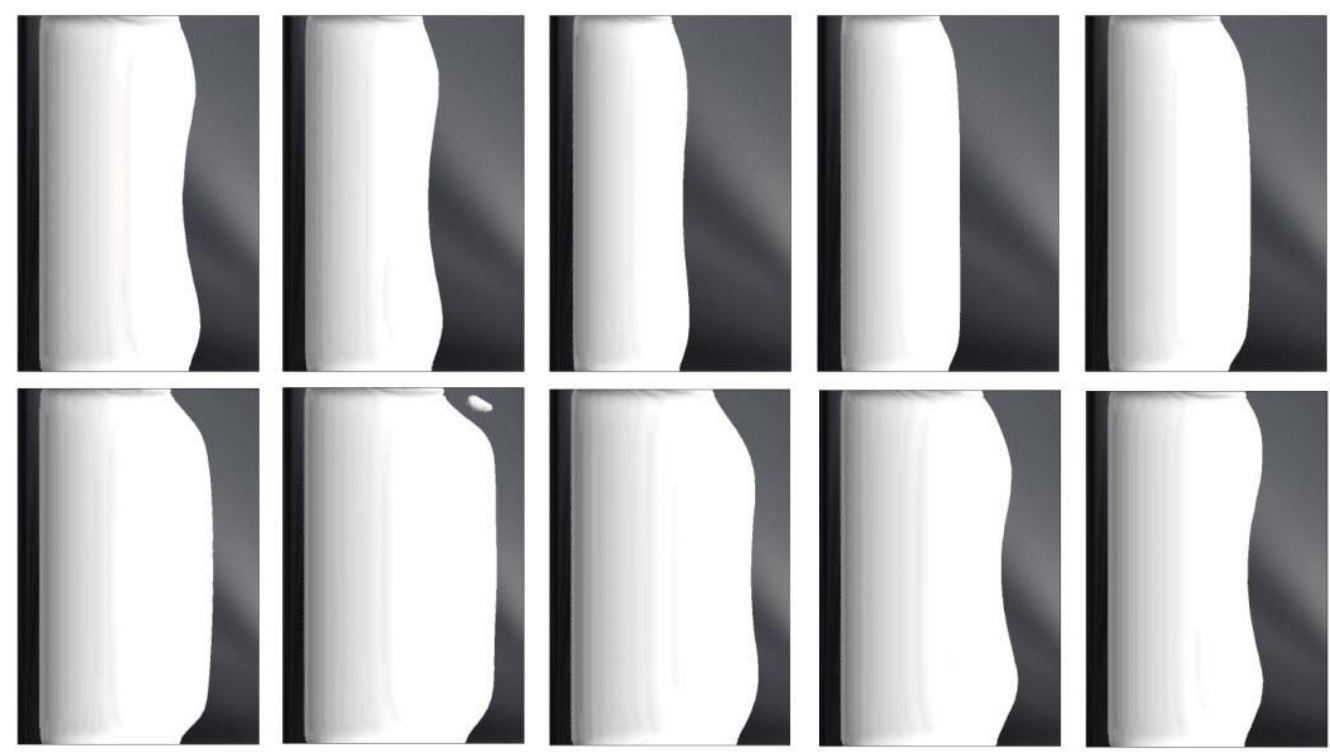

Figure 9: Partial cavity oscillation. CFD analysis, $\alpha=0^{\circ}, \sigma=1.8$

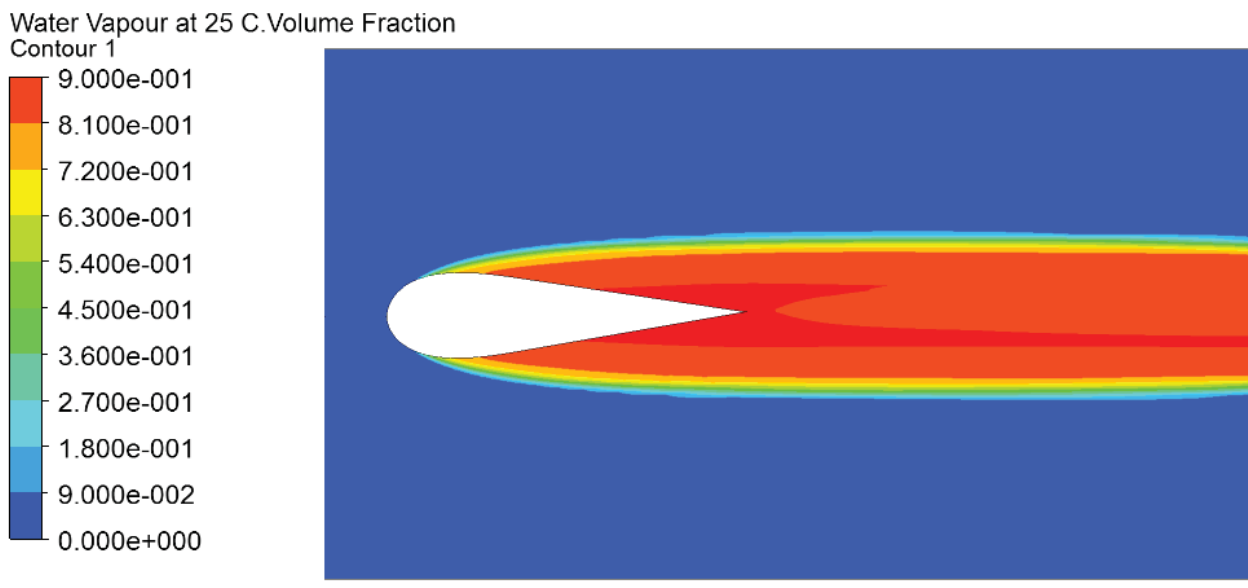

Figure 10: Distribution of void fraction in the symmetry plane during steady supercavitation regime. CFD, $\alpha=0^{\circ}, \sigma=1.5$ 

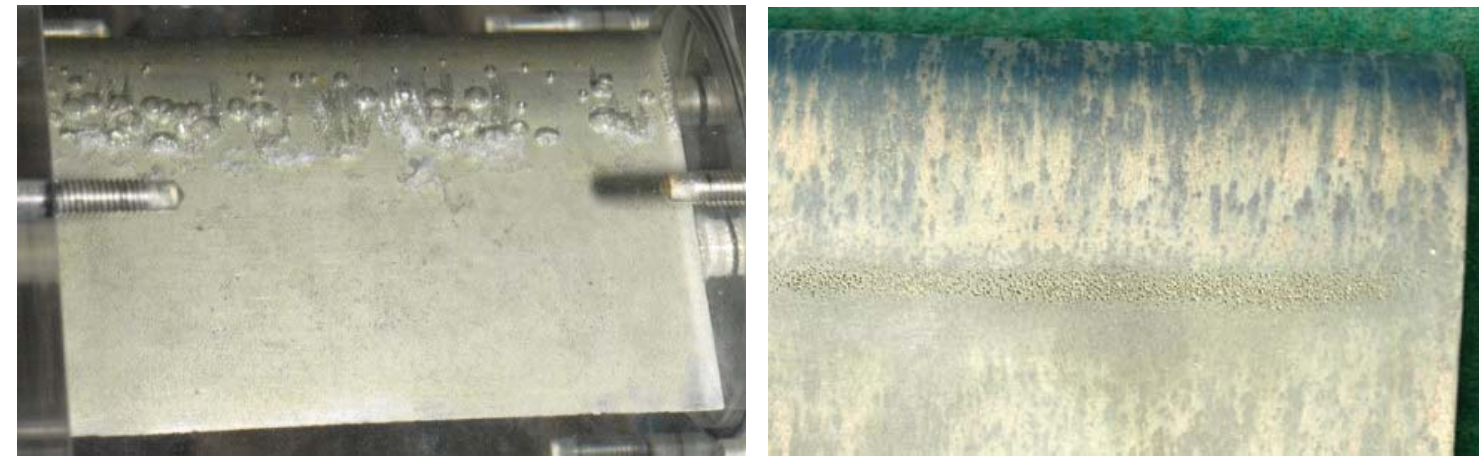

Figure 11: Individual bubbles just before the first collapse and a narrow stripe of pits on hydrofoil upper side. $\alpha=-1^{\circ}, \sigma=1.93$
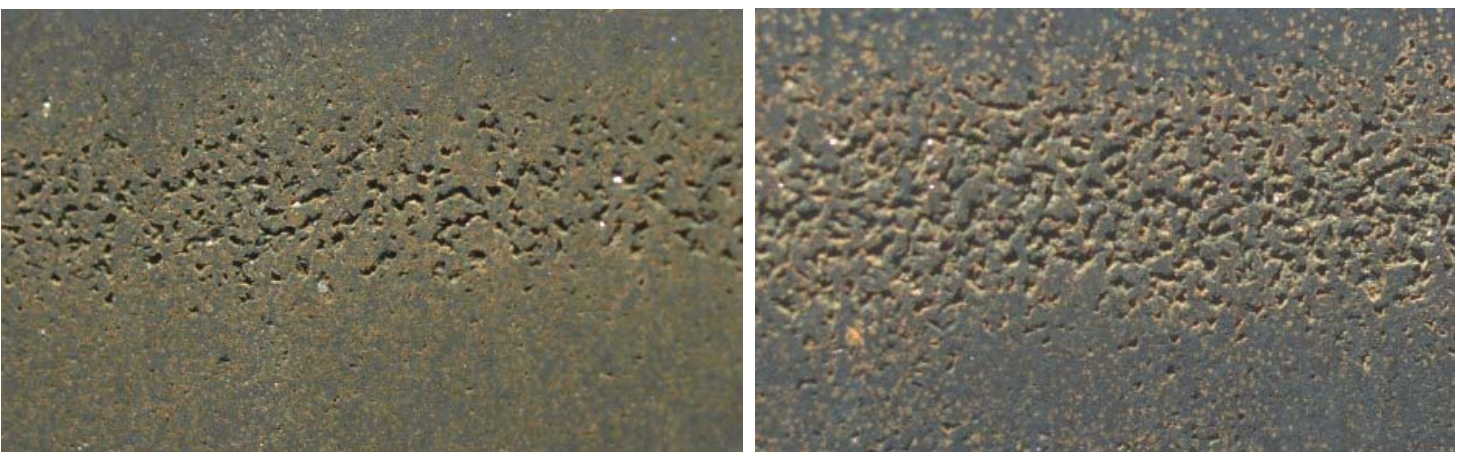

Figure 12: Detail of cavitation pits on the surface of hydrofoil made of cast iron after 63 and 120 hours. $\alpha=-1^{\circ}, \sigma=1.93$

The results of the pitting tests were compared with the results of the numerical modelling of the bubble dynamics and the evaluation of the erosion potential of bubble collapses. To avoid the influence of the side boundary layers, the results were evaluated at midspan of the hydrofoil. Figure 15 shows the pressure distribution along the upper and the lower surfaces of the hydrofoil in the symmetry plane. Here the variable $x$ means the blade chord length; $x=0$ represents the leading edge and $x=1$ represents the trailing one. The dynamics of two bubbles originating from the smallest and the largest cavitation nuclei along the upper mid-span streamline can be seen in Fig. 16. Though there is a great difference between the initial radii of the presented nuclei, there is a very similar resulting bubble size before the first collapse, between 2 and $3 \mathrm{~mm}$ in diameter. The maximum dimension of the observed bubbles determined from the photographs (Fig. 11) is about $4-5 \mathrm{~mm}$. If we take into account a deformation of the largest observed bubbles in the vicinity of the solid surface, we get a very good agreement of the mean radius of these bubbles. The cumulative erosion potential of all bubbles of the same size along the upper and lower streamlines is shown in Fig. 17. The erosion potential of the first collapses is several orders higher than that for the successive rebounds. All the calculated first collapses are located between 26 and $28.5 \%$ of the chord length on the upper side of the blade and between 32 and $33 \%$ of the chord length on the lower side. If we take into account, that the real flow past the blade in the cavitation tunnel is influenced by the turbulent structures, especially on the lower surface with a less stable boundary layer, the CFD analysis agrees very well with the location of pits on the blade surface. They are located between 27 and $32 \%$ of the chord length on the upper side of 
the blade and between 32 and $37 \%$ of the chord length on the lower side. The narrow stripes of pits on both sides of the hydrofoil confirmed our observations that for the cavitation number of 1.93 the cavitation region is really steady and has a shape of the stripe, which is equally wide in the spanwise direction.

We also tried to model numerically the bubble dynamics in the steady supercavitation regime, which corresponds to the Figures 8 and $10\left(\alpha=0^{\circ}, \sigma=1.5\right)$. Figure 18 shows the pressure distribution along the upper surface of the hydrofoil in the symmetry plane (the pressure distribution along the lower surface is the same). The dynamics of two bubbles originating from the smallest and the largest cavitation nuclei along the upper mid-span streamline can be seen in Fig. 19. Theoretically the cavitating bubbles can reach about $20 \mathrm{~mm}$ in diameter before the first collapse, but it is in contrary with the observations, in which the largest bubbles about $7 \mathrm{~mm}$ in diameter were found. The reason is that the void fraction inside the cavitation region is very high (Fig. 10 and 20) and the bubbles strongly influence each other, which is in contrary with the assumptions of the presented cavitation erosion model.

\section{CONCLUSIONS}

This paper presents some results rising from the experiments carried out in the cavitation tunnel built in the SIGMA Research and Development Institute. Though the main objective of the experimental research was to validate the cavitation erosion model, very interesting cavitation phenomena were visualized during the extensive tunnel testing. The detailed analysis of these phenomena (especially the partial and the transitional cavity oscillations as well as the unsteady supercavitation regimes at different incidence angles) will be the topic of the next research, but it requires the additional experimental equipment, especially two simultaneous high-speed video cameras. Nevertheless very narrow stripes of the pits on both sides of the hydrofoil confirmed our observations, that for the cavitation number of 1.93 the cavitation region is really steady and has a shape of the stripe, which is equally wide in the spanwise direction.

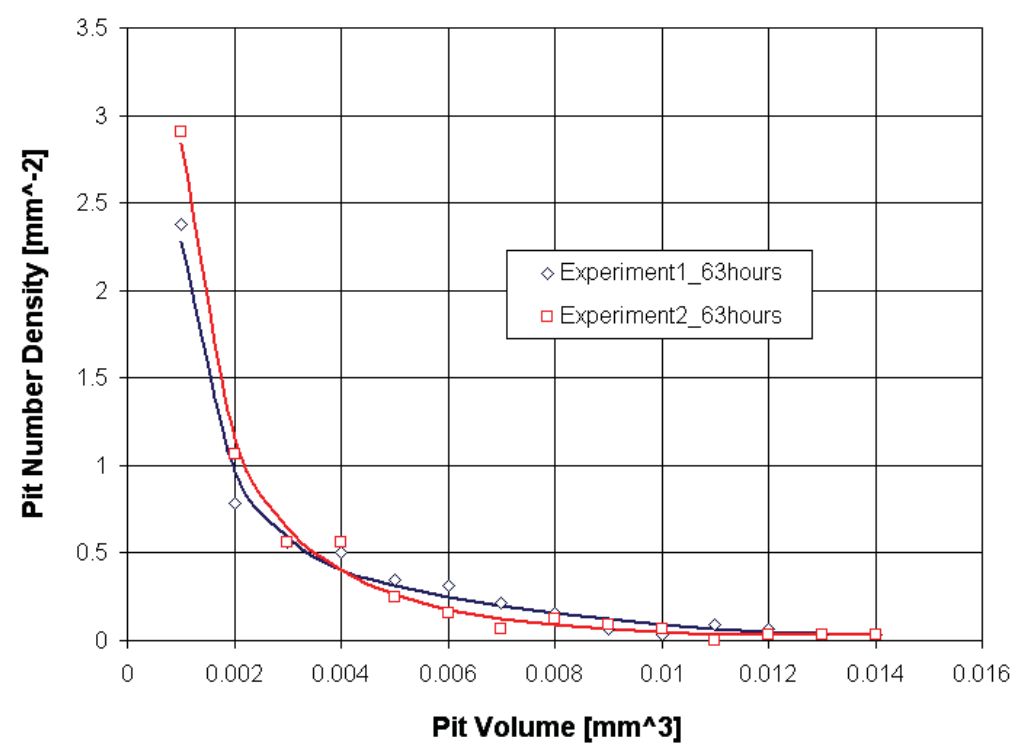

Figure 13: Size distribution of pits in cast iron hydrofoil after 63 hours and repeatability of measurements 

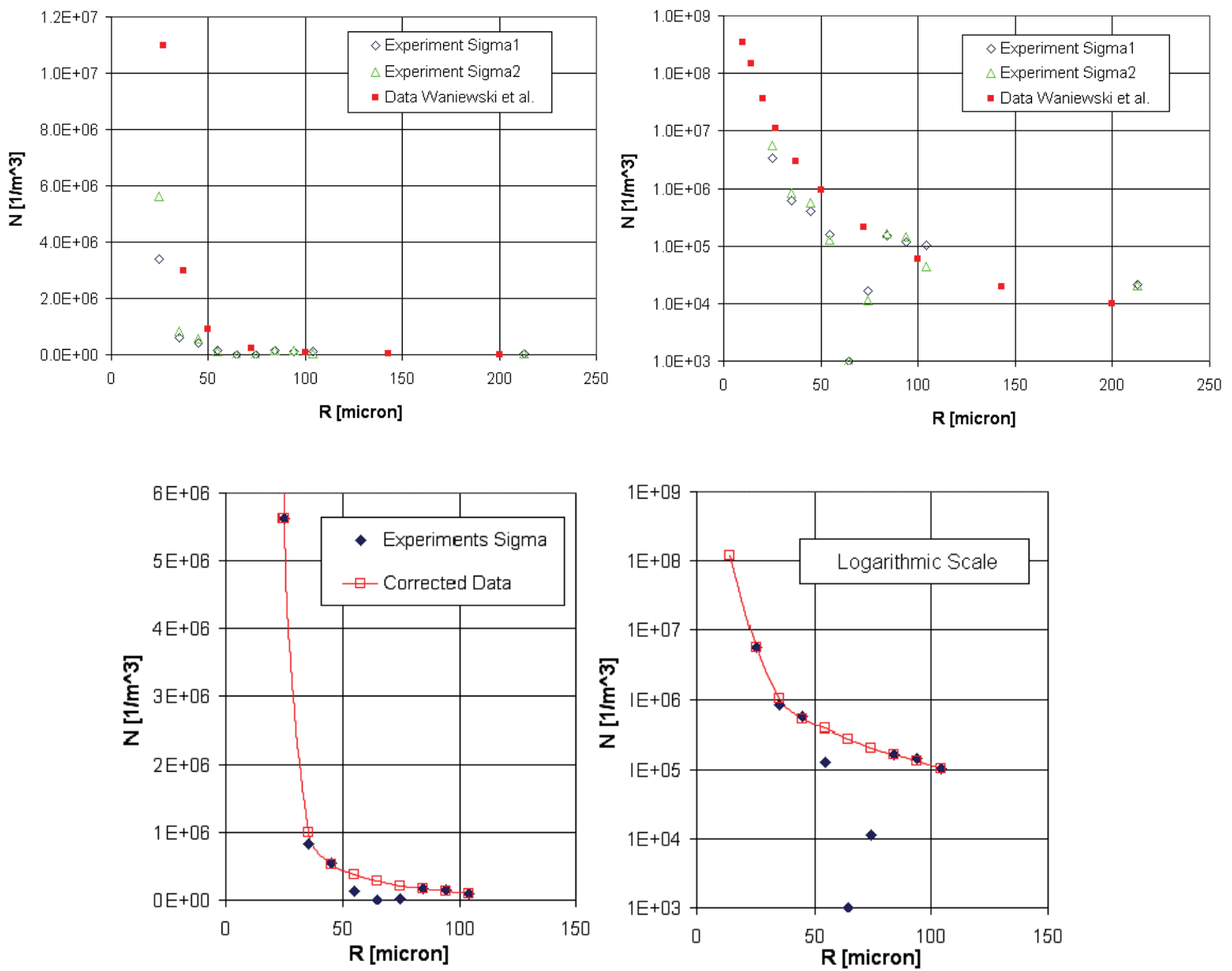

Figure 14: Inlet nuclei distribution measured by acoustic bubble spectrometer

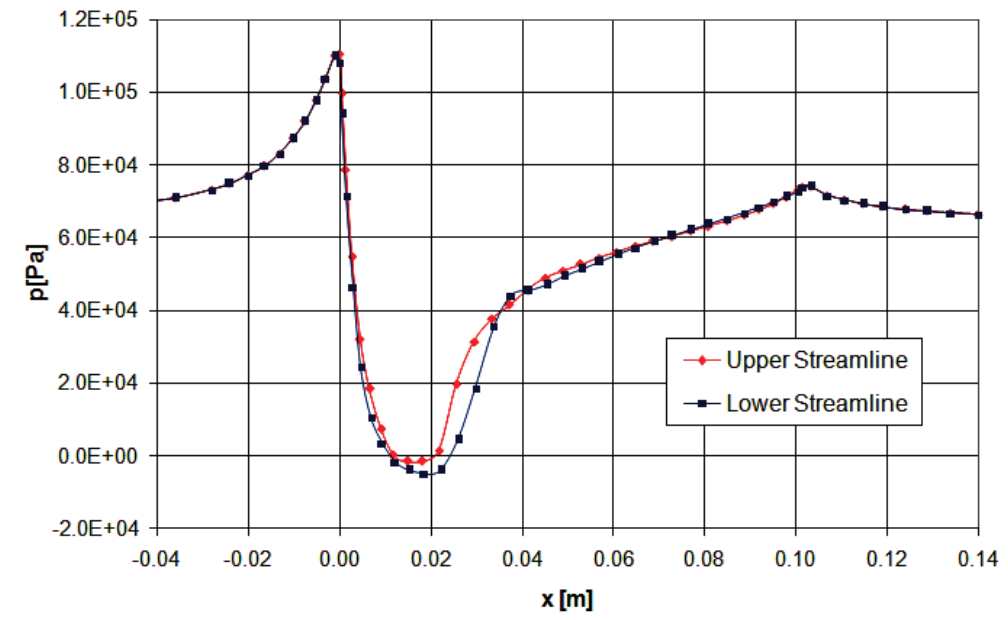

Figure 15: Pressure distribution along the upper and lower surfaces of the hydrofoil in the symmetry plane. $\alpha=-1^{\circ}, \sigma=1.93$ 


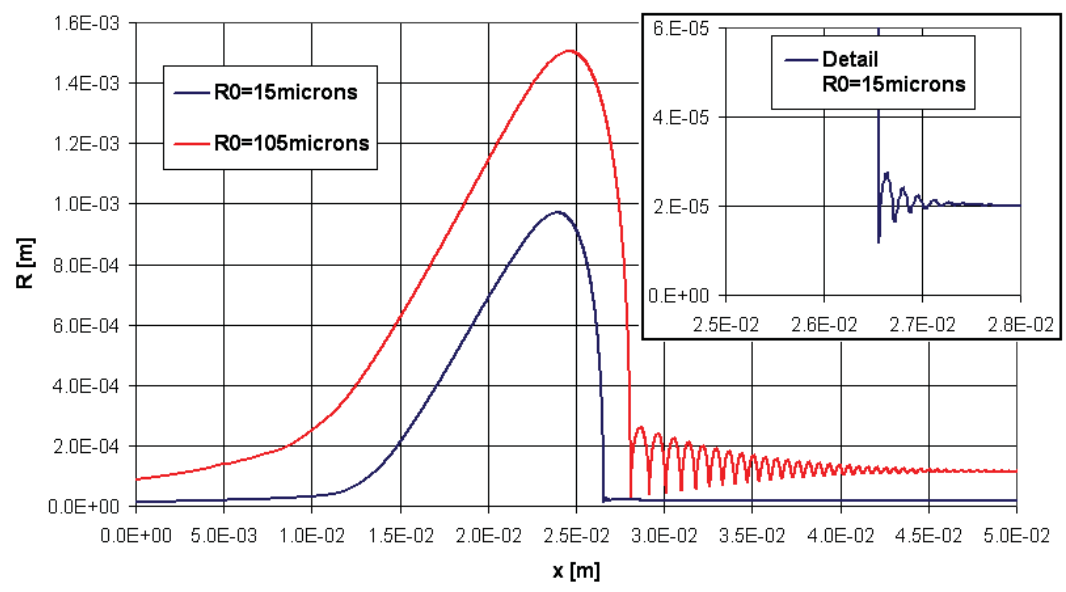

Figure 16: Dynamics of two bubbles originating from the smallest and largest cavitation nuclei along the upper mid-span streamline.

$\alpha=-1^{\circ}, \sigma=1.93$
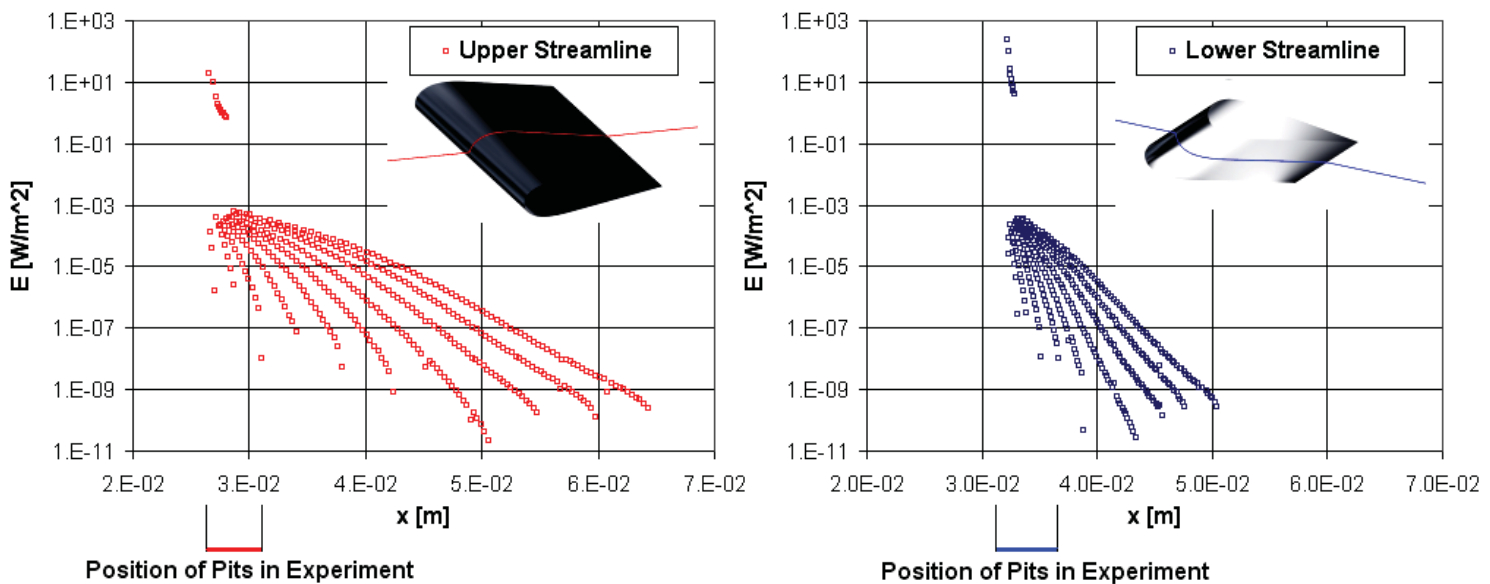

Position of Pits in Experiment

Position of Pits in Experiment
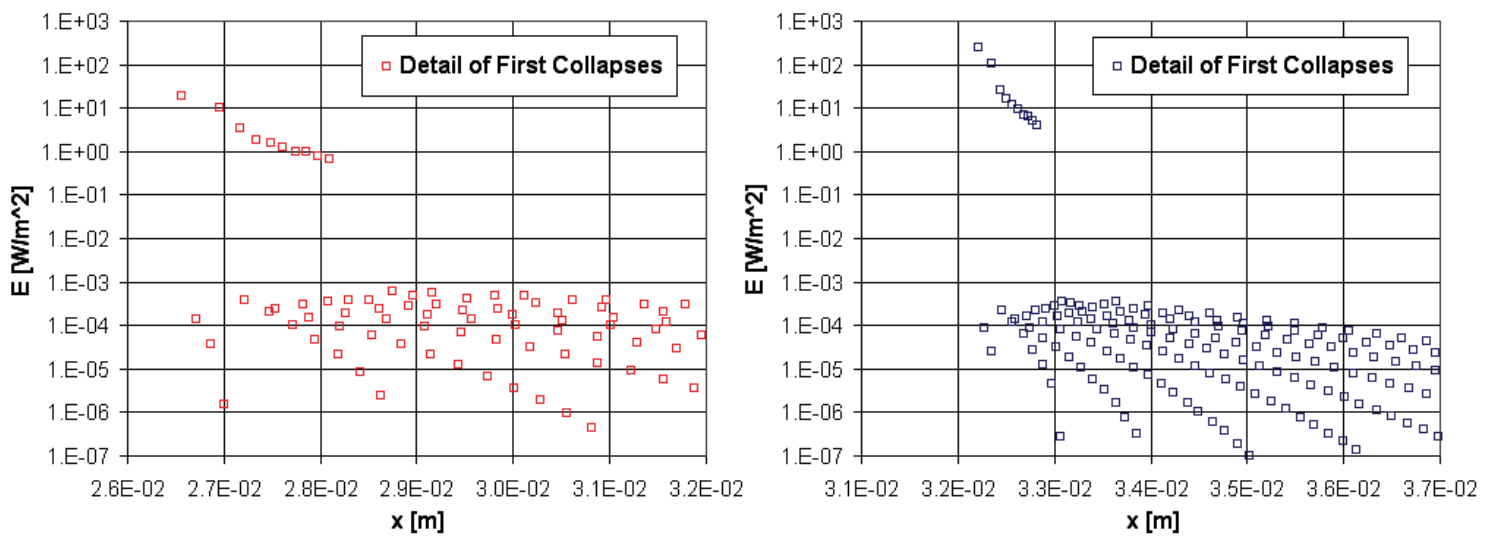

Figure 17: Cumulative erosion potential of all bubbles of the same size along the upper and lower streamlines. $\alpha=-1^{\circ}, \sigma=1.93$

The presented cavitation erosion analysis shows encouraging agreement of the theoretical results and the observations. The calculated position and energy of the first collapses corresponds well with the measured position and size distribution of the pits as 
well as with the measured inlet nuclei distribution. Of course, a lot of restricting considerations have been accepted in the numerical model. That is why it is supposed to be used for the assessment of the cavitation erosion in the hydraulic machine when the erosion is caused by collapses of the individual cavitation bubbles which do not strongly interact.

\section{ACKNOWLEDGMENTS}

This work has been supported by the Czech Science Foundation under the grant no. 101/10/1428 "Collapse and fission mechanisms of ultrasonically excited cavitation bubbles near a solid boundary in Newtonian liquid" and by the Ministry of Education of the Czech Republic under the grant no. 4674788501 "Optimization of Machine Properties in Interaction with Working Processes and Human Aspects". The authors are grateful to Dr. Patrik Zima from the Institute of Thermomechanics of the Academy of Sciences of the Czech Republic for his kind help with the numerical modelling of the cavitation erosion as well as preparing the experiments.

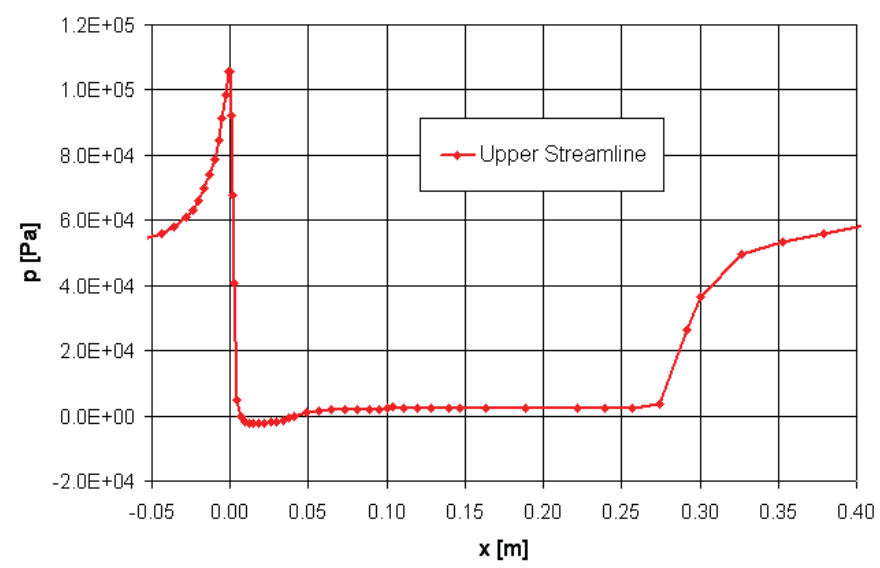

Figure 18: Pressure distribution along the upper surface of the hydrofoil in the symmetry plane. $\alpha=0^{\circ}, \sigma=1.5$

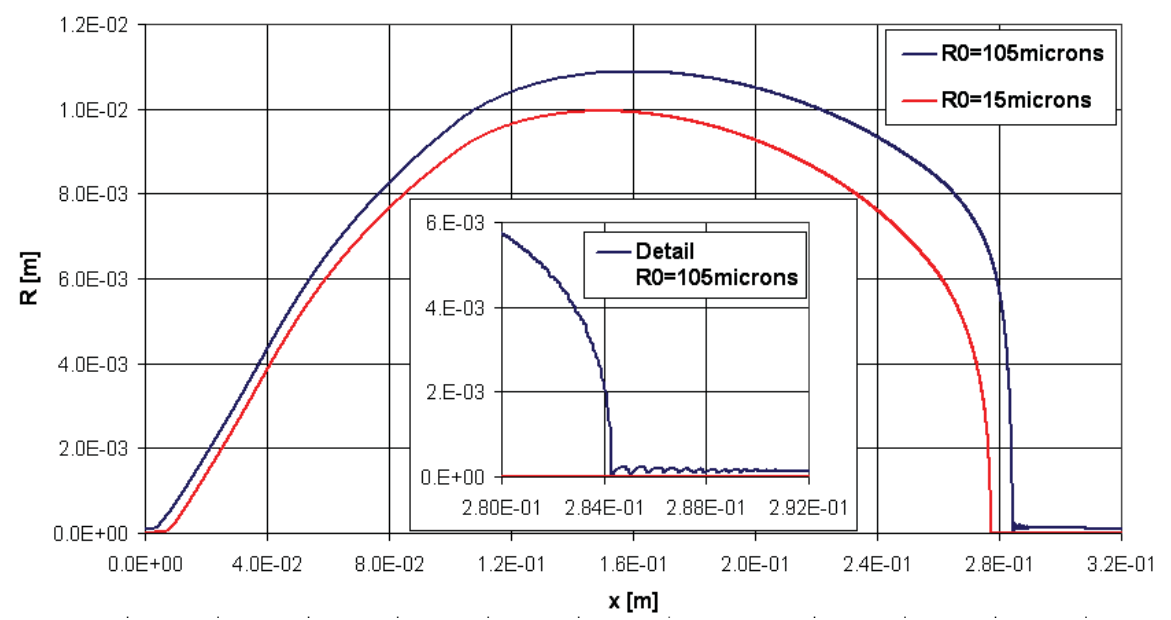

Figure 19: Dynamics of two bubbles originating from the smallest and largest cavitation nuclei along the upper streamline. $\alpha=0^{\circ}, \sigma=1.5$ 


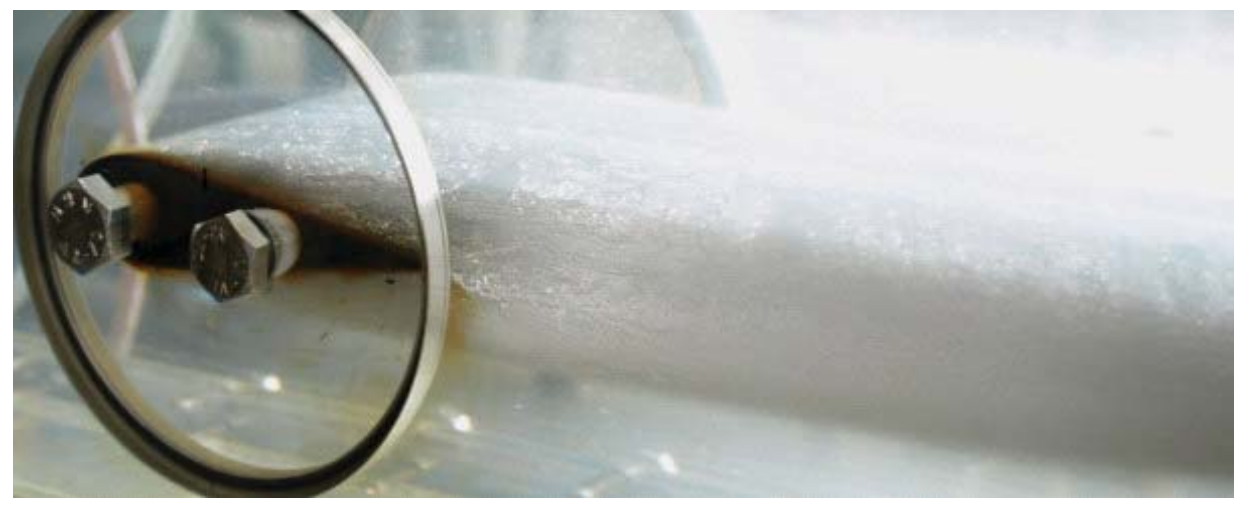

Figure 20: Steady supercavitation regime. Experiment, short time exposure. $\alpha=0^{\circ}, \sigma=1.5$

\section{References}

[1] Sedlář M., Maršík F., Šafařik P.: Numerical Investigation of Blade and Tip Clearance Geometry Influence on Tip Clearence and Tip Vortex Cavitations, Proc. 5th ISAIF, Vol.1, 2001, pp. 237-245

[2] Sedlář M., Doubrava V., Zbořil, J.: Numerical Analysis of Cavitation Phenomena in Pump Impeller at Different Values of Suction Head, Proc. 17th Int. Conf. Hydro-Turbo 2004, 2004, pp. 127-132

[3] Sedláŕ M., Bajorek M., Šoukal J.: Investigation of Cavitation Phenomena in Suction Part of Radial-Flow Multistage Pump, Proc. ETC7, 2007, pp. 785-794

[4] Sedlář M., Šputa O., Komárek M.: Numerical Modelling of Cavitation Properties of Mixed-Flow Pump, Proc. WIMRC 3rd Int. Cavitation Forum, 2011

[5] Sedlár M.: Numerical Analysis of Three-Dimensional Turbulent Flow in the Pump Impeller at Partial Load, Proc. 10th Conf. on Num. Methods in Laminar and Turbulent Flow, Univ. of Swansea, 10, 1997, pp. 97-104

[6] Sedlář M., Maršík F., Šafařík P.: Modelling of Cavitated Flows in Hydraulic Machinery Using Viscous Flow Computation and Bubble Dynamics Model, Proc. Fluid Dynamics 2000, 2000, pp. 107-110

[7] Sedlář M., Maršík F., Šafařík P.: Numerical Analysis of Cavitated Flows in MixedFlow Pump Impellers. Proc. 3rd Int. Conference on Transport Phenomena in Multiphase Systems, Heat 2002, 2002, pp. 461-466

[8] Sedlář M., Zima P., Němec T., Maršík F.: Analysis of Cavitation Phenomena in Water and its Application to Prediction of Cavitation Erosion in Hydraulic Machinery, Proc. 15th Int. Conf. ICPWS, 2008

[9] Zima P., Sedlář M., Maršík F.: Bubble Creation in Water with Dissolved Gas: Prediction of Regions Endangered by Cavitation Erosion, In: Water, Steam, and Aqueous Solutions for Electric Power, Advances in Science and Technology, Kyoto, Maruzen Co. Ltd., 2005, pp. 232-235

[10] Zima P., Sedláŕ M., Muller M.: Modeling collapse aggressiveness of cavitation bubbles in hydromachinery, Proc. 7th Int. Symposium on Cavitation, CAV 2009, 2009

[11] Menter F.R., Kuntz M. and Bender R.: A Scale-Adaptive Simulation Model for Turbulent Flow Prediction, AIAA paper 2003-0767, 2003 
[12] Watanabe S., Konishi Y., Nakamura I., Furukawa A.: Experimental Analysis of Cavitating Behavior around a Clark Y Hydrofoil, Proc. WIMRC 3rd Int. Cavitation Forum, 2011

[13] Waniewski T.A., Hunter C., Brennen C.E.: Bubble Measurements Downstream of Hydraulic Jumps, Int. J. Multiphase Flow, Vol. 27, 2001, pp. 1271-1284 